\title{
Ligand conjugated lipid-based nanocarriers for cancer theranostics
}

\author{
Rahul Kumar ${ }^{1}$, Daphika Dkhar $\mathrm{S}^{1}$, Rohini Kumari ${ }^{1}$, Supratim Mahapatra ${ }^{1}$, ${ }^{*}$ Divya $^{1}$, \\ Vikash Kumar Dubey ${ }^{1}$, and Pranjal Chandra ${ }^{1}$ \\ ${ }^{1}$ Indian Institute of Technology BHU Varanasi
}

February 28, 2022

\begin{abstract}
Cancer is one of the major health-related issues affecting the population worldwide and subsequently accounts for the secondlargest death. Genetic and epigenetic modifications in oncogenes or tumor suppressor genes affect the regulatory systems that lead to the initiation and progression of cancer. Conventional methods, including chemotherapy/radiotherapy/appropriate combinational therapy and surgery, are being widely used for theranostics of cancer patients. Surgery is useful in treating localized tumors, but it is ineffective in treating metastatic tumors, which spread to other organs and result in a high recurrence rate and death. Also, the therapeutic application of free drugs is related to substantial issues such as poor absorption, solubility, bioavailability, high degradation rate, short shelf-life, and low therapeutic index. Therefore, these issues can be sorted out using nano lipid-based carriers (NLBCs) as promising drug delivery carriers. Still, at most, they fail to achieve site targeted drug delivery and detection. This can be achieved by selecting a specific ligand/antibody for its cognate receptor molecule expressed on the cancer cell surface. In this review, we have mainly discussed the various types of ligands used to decorate NLBCs. A list of the ligands used to design nanocarriers to target malignant cells specifically has been extensively undertaken, and the approved ligand decorated lipid-based nanomedicines with their clinical status has been explained in tabulated form to provide a wider scope to the readers regarding ligand coupled NLBCs.
\end{abstract}

\section{Hosted file}

Manuscript_Final.docx available at https://authorea.com/users/443410/articles/558102-ligandconjugated-lipid-based-nanocarriers-for-cancer-theranostics 